\title{
Nonlinear analysis of BPSK optical phase locked loops using MATLAB and Simulink
}

\author{
Ziyang Chen ${ }^{1, a^{*}}$, Yunpeng $\mathrm{Hu}^{2, \mathrm{~b}}$ and Lu Liü, \\ 1,2 Zhengzhou Information Science and Technology Institute, Department of Communication \\ Engineering, Zhengzhou, Henan 450001, P.R. China \\ ${ }^{3}$ Peking University, School of Electronics Engineering and Computer Science, RM2046, Science \\ Building Two, Haidian District, Beijing 100871, P.R. China \\ axxgc_czy@163.com, bhyp1978@126.com, ${ }^{c}$ luliu@pku.edu.cn
}

Keywords: Optical phase-locked loop, Balanced Loop, Costas Loop, MATLAB Simulation Abstract. Optical phase locked loops (OPLLs) plays a vital role in coherent detection of optical communication systems. To simplify the analysis of OPLLs with complex structure, two different Simulink models are presented to have a vivid observation of nonlinear BPSK OPLLs. Simulation results show that both balanced OPLL and Costas OPLL can recover data information without residual carrier while Costas OPLL shows advantage in system robustness and complexity.

\section{Introduction}

Compared with conventional intensity modulation/direct detection (IM/DD), coherent detection has its advantages in improved receiver sensitivity and enhanced bandwidth selectivity [1]. Also, as information can be carried on both amplitude and phase of the carrier, coherent detection allows greater flexibility in modulation formats and optimizes the spectral efficiency [2].

Optical phase locked loop (OPLL) plays a vital role in the coherent optical communication. A thorough analysis of nonlinear OPLL is a difficult task, to simplify the mathematical model and have a vivid observation of the real-time loop functioning, simulation methods are widely used. There have been works about basic electric communication systems and BPSK Costas loop in MATLAB simulation [3], but simulation concerning about optical signals hasn't been discussed in detail. Having the special operating forms of optical signals taken into consideration, this paper proposes two novel OPLL simulation models for coherent optical communication to have a guide into the relevant research. The simulation is based on the BPSK modulation receiver and here we'll dig into a balanced OPLL and a Costas OPLL to make a comparison between the two loops ${ }^{1}$.

\section{OPLL basic structure and concepts}

Optical phase locked loop (OPLL) shares the similar loop design theory as electrical PLLs but the physical devices are different. A brief block diagram of OPLL is presented in Fig. 1. OPLL uses a laser as the controlled oscillator rather than transistor-based oscillators or crystals as in electrical PLL. Also, photodetectors are normally used as phase detectors.

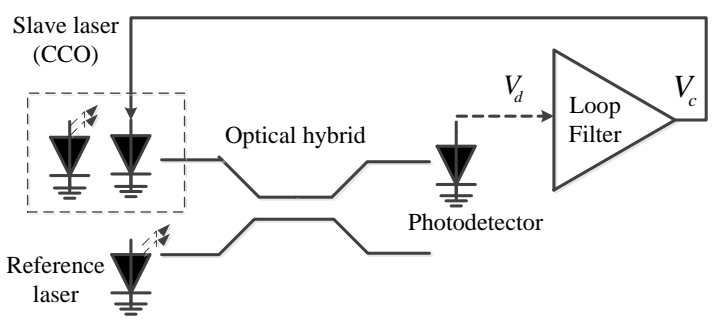

Fig. 1 Basic block diagram of OPLL

After beating the two lasers on the photo detector, the output photocurrent can be written as [4]

$$
I_{P D} \propto\left|E_{R} e^{j\left(2 \pi f_{R} t+\theta_{R}\right)}+E_{L} e^{j\left(2 \pi f_{L} t+\theta_{L}\right)}\right|^{2}=\left|E_{R}\right|^{2}+\left|E_{L}\right|^{2}+2\left|E_{R}\right|\left|E_{L}\right| \cos (\Delta \theta(t)) .
$$

\footnotetext{
${ }^{1}$ This work is supported by National Science Foundation under GrantNo. 61501517.
} 
In Eq.1, $f_{R}$ and $f_{L}$ denotes the frequency of reference laser and slave laser respectively. $E_{R}, \theta_{R}$ and $f_{R}$ are the electric field amplitude, phase, and frequency of the reference laser, while $E_{L}, \theta_{L}$ and $f_{L}$ are the counterparts of slave laser. Based on the structure above, two kinds of OPLL Loops are simulated and analyzed using MATLAB Simulink in the following chapters.

\section{Simulink models for OPLL}

The balanced loop uses $180^{\circ}$ optical hybrid mixer and two balance detectors as PDs. The corresponding MATLAB and Simulink model for balanced OPLL [5] is shown in Fig.1. Since the BPSK modulation suppresses its carrier information, in order to provide a residual carrier, a square module is applied to recover the carrier information. In this way, an extra frequency divider module is needed since the loop is locked in frequency $2 \omega_{s}$.

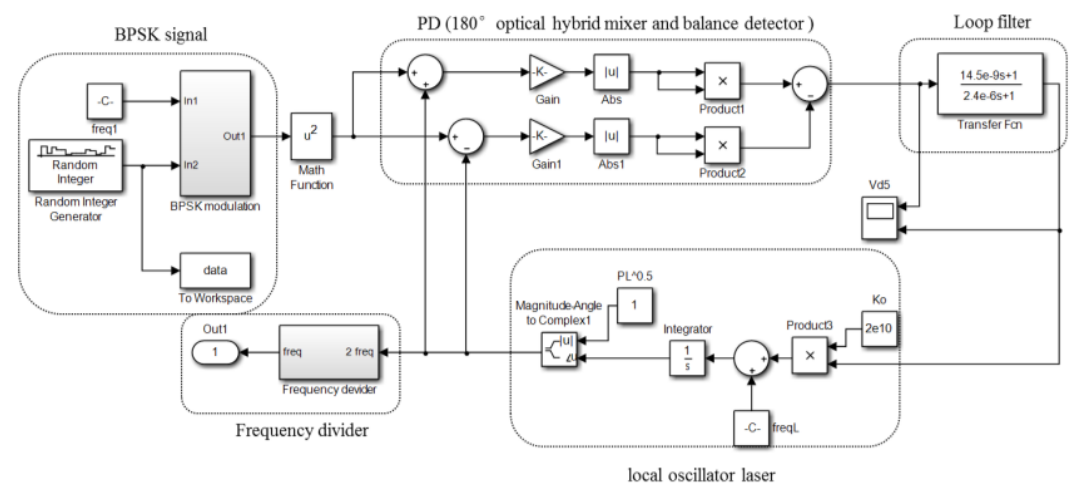

Fig 2. Balanced OPLL model for BPSK signal tracking

Costas loop differs from the balanced loop basically in their functions between control voltage and phase error. Since Costas OPLL can combine the information from both I/Q signals, it doesn't require for the residual carrier and can be used to recover the original BPSK data. The corresponding model for Costas OPLL is shown in Fig. 2.

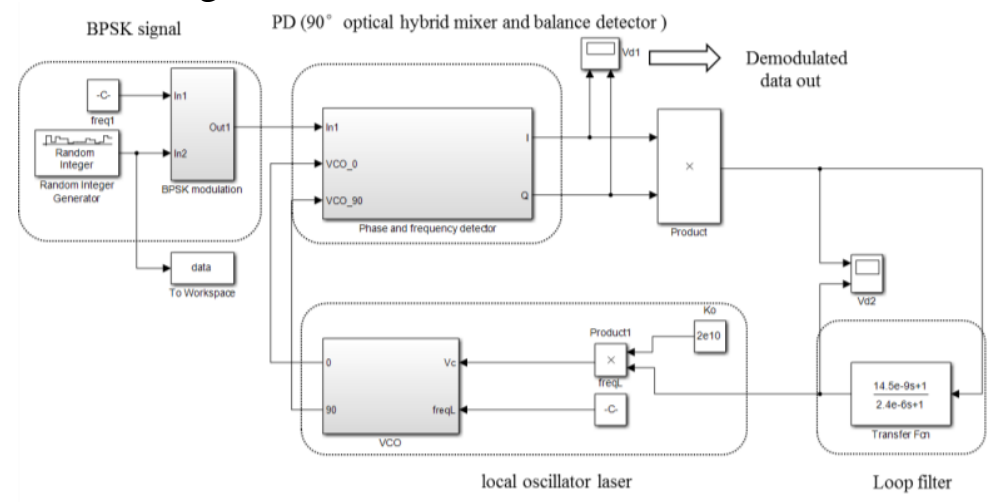

Fig.3 Costas loop simulation model for BPSK signals

The integrated block of phase and frequency detector in Costas OPLL consists two parts, namely the $90^{\circ}$ optical hybrid mixer and the balanced detector. Based on the optical operation rule, they are constructed as follows.
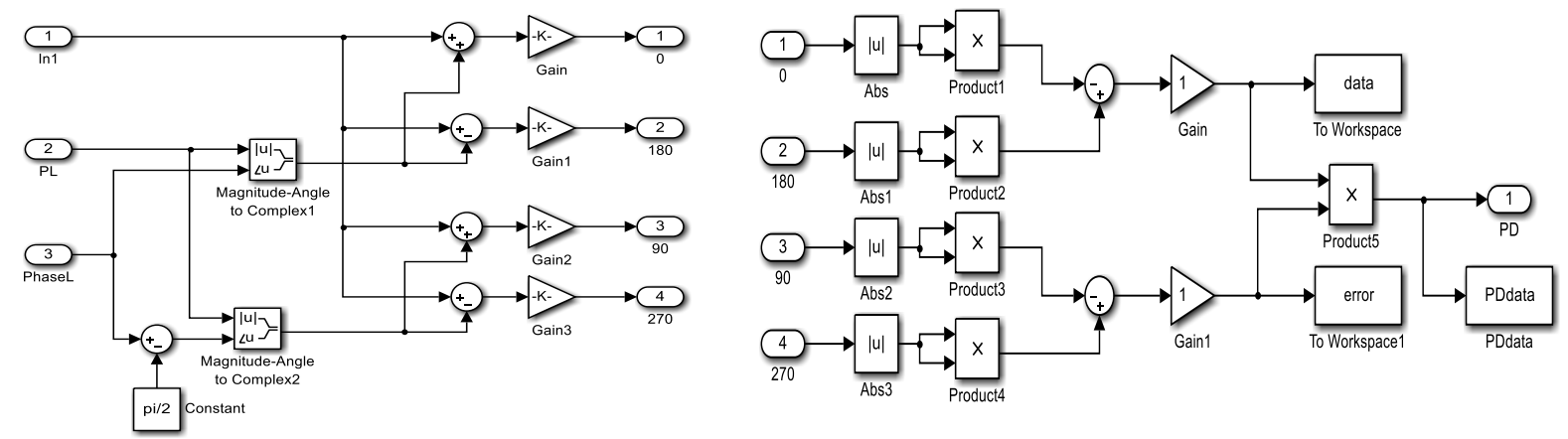

Fig.4 $90^{\circ}$ optical hybrid mixer (left) and balanced detector (right) 
The phase shift $\pi / 2$ is introduced to insert $e^{-\frac{\pi}{2} j}$ in the quadrature branch. The Gains are set as $1 / \sqrt{2}$. The four outputs from $90^{\circ}$ optical hybrid mixer are sent to the balance detector to produce the error voltage $V_{d}$.

\section{Measurement and Characterization}

Table 1 gives a summary of parameters for the OPLL simulations. Note that OPLL has a vast ratio of oscillator frequency to loop bandwidth [6]; the loop is working under Gbps while the loop bandwidth is ranged in $\mathrm{MHz}$.

Table 1 Parameters for the OPLL simulations

\begin{tabular}{c|ccc}
\hline Parameters & Value & Unit & Descriptions \\
\hline$w_{S}$ & $2 \pi \times\left(10^{11}+10^{6}\right)$ & {$[\mathrm{rad} / \mathrm{s}]$} & Signal frequency \\
$w_{L o}$ (for balanced OPLL) & $2 \pi \times 2 \times 10^{11}$ & {$[\mathrm{rad} / \mathrm{s}]$} & Local laser frequency \\
$w_{L o}$ (for Costas OPLL) & $2 \pi \times 1 \times 10^{11}$ & {$[\mathrm{rad} / \mathrm{s}]$} & Local laser frequency \\
$K_{0}$ & $2 \times 10^{10}$ & {$[\mathrm{rad} / \mathrm{s} / \mathrm{V}]$} & VCO sensitivity \\
$R_{b}$ & $1 \times 10^{9}$ & {$[\mathrm{bits} / \mathrm{s}]$} & BPSK data rate \\
$H(s)$ & $14.5^{*} 10^{-9} S+1$ & & Transfer function \\
\hline
\end{tabular}

The bode diagram of magnitude and Phase response of $\mathrm{H}(\mathrm{s})$ is shown in Fig. 5 as follows.

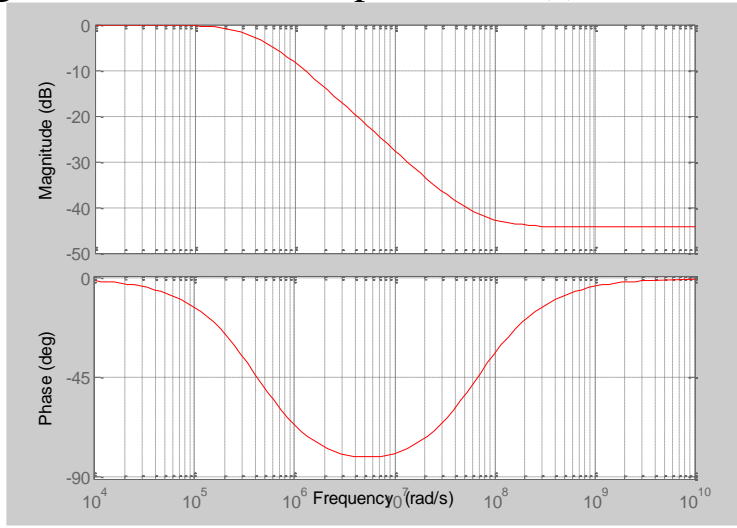

Fig. 5 Bode Diagram of OPLL loop filter

Seen from Fig.5, the loop band width is in MHz and the error voltage will pass this loop while the carrier frequency is suppressed. The scope displays the dynamic variation of error voltage and control voltage as shown in Fig. 6. When the loop has fallen into lock, the control voltage will remain stable later on.
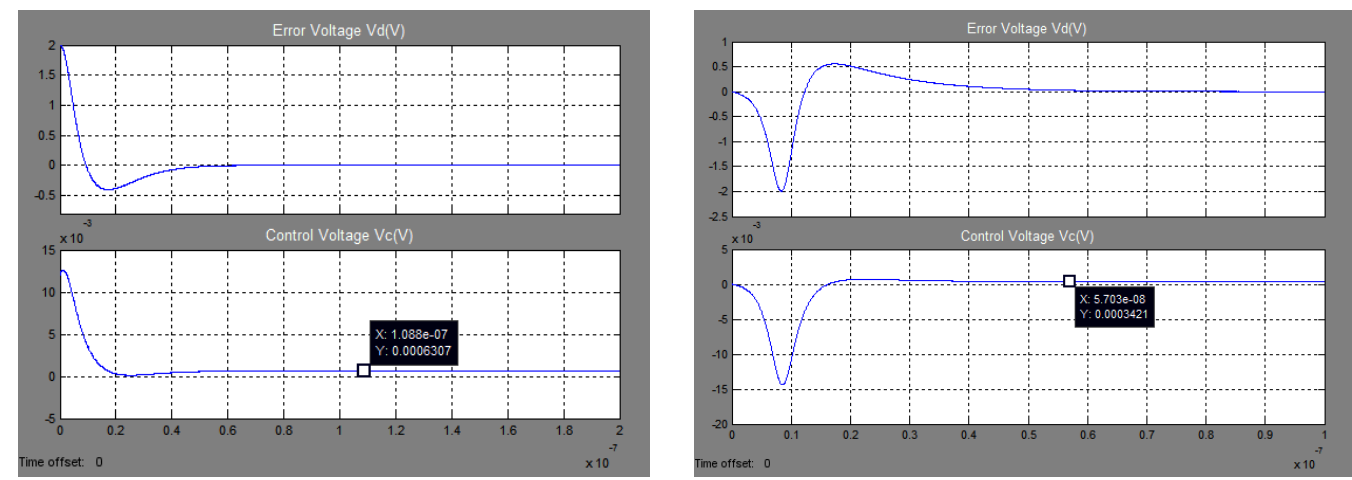

Fig.6 Error voltage and control voltage of balanced OPLL (left) and Costas OPLL (right)

Due to the different loop construction, the local laser frequency of balanced OPLL is set as $2 \pi \times 2 \times 10^{11} \mathrm{rad} / \mathrm{s}$, while it is $2 \pi \times 1 \times 10^{11} \mathrm{rad} / \mathrm{s}$ for Costas OPLL. From the data cursor appended in the picture, the value of stable control voltage equals $6.307 \times 10^{-4} \mathrm{~V}$ and $3.421 \times 10^{-4}$ respectively for the two loops. Analysis of the loop states can be made as follows. As for Balanced OPLL, we have: 


$$
\omega_{c 1}=\omega_{L o}+K_{o} V c=2 \pi \times 2 \times\left(10^{11}+10^{6}\right)=2 \omega_{s} .
$$

Therefore the initial signal frequency $\omega_{s}$ can be obtained after the frequency divider. The process of squaring and dividing are non-linear which will induce harmonic components and increase the system complexity. Take similar analyze for Costas OPLL, it is as below. The loop is in locked since the final frequency output from VCO equals signal frequency.

$$
\omega_{c 2}=\omega_{L o}+K_{o} V c=2 \pi\left(10^{11}+10^{6}\right)=\omega_{s}
$$

The output of I/Q branch in Costas loop depicts BPSK data sequences as shown in Fig.7. Here we present the data sequence recorded from MATLAB workspace for comparison:

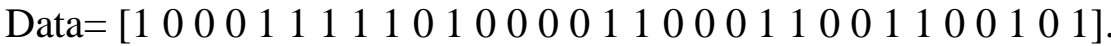

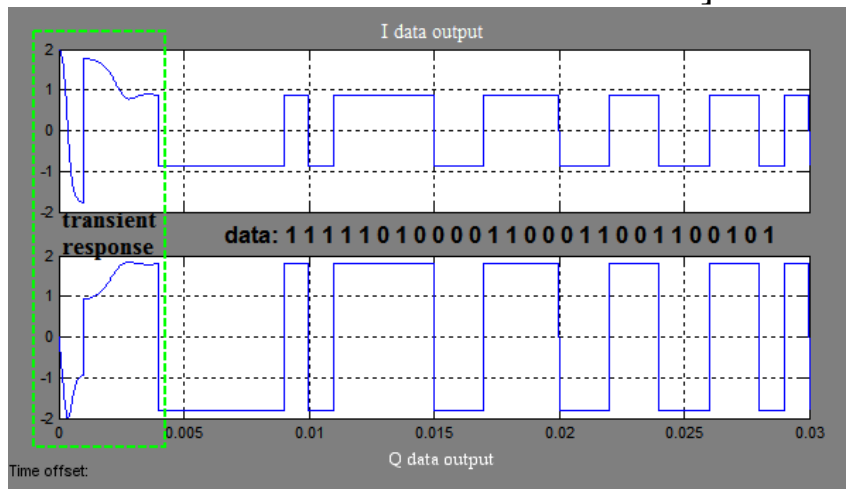

Fig.7 Demodulated data of BPSK signal in Costas loop

Seen from the figure, the high levels output from I/Q represent data ' 0 ', and the low levels represent data ' 1 '. If transient response is neglected, the Costas loop can recover the data accurately.

\section{Conclusions}

In order to implement the synchronization of none-residual carrier optical signals, non-linear OPLLs are required in coherent optical communication systems. Here we provide a novel method to analyze the structure and operating principles of non-linear OPLLs based on MATLAB Simulink. Both balanced OPLL and Costas OPLL are proposed in the demodulation of BPSK signal. Due to that the balanced loop is a linear loop; it requires an extra frequency square module and frequency divider module to ensure the loop fall into lock which will increase the system cost and complexity. Costas loop can avoid this disadvantage by utilizing the $90^{\circ}$ optical hybrid and I/Q detector. In future, we will make some numerical analysis and comparisons for the two OPLLs in detail.

\section{References}

[1] Kazovsky, Leonid G. Balanced phase-locked loops for optical homodyne receivers: Performance analysis, design considerations, and laser linewidth requirements. Lightwave Technology, Journal of 4.2 (1986): 182-195.

[2] Ip, Ezra, et al. Coherent detection in optical fiber systems. Optics Express 16.2 (2008): 753-791.c.

[3] Kuznetsov, N. V., et al. BPSK Costas loop: simulation of nonlinear models in MATLAB Simulink. Ultra Modern Telecommunications and Control Systems and Workshops (ICUMT), 2014 6th International Congress on. IEEE, 2014.

[4] Bloch, Eli, et al. A 1-20-GHz All-Digital InP HBT Optical Wavelength Synthesis IC. Microwave Theory and Techniques, IEEE Transactions on 61.1 (2013): 570-580.

[5] Tranter, William, Ratchaneekorn Thamvichai, and Tamal Bose. Basic Simulation Models of Phase Tracking Devices Using MATLAB. Synthesis Lectures on Communications 4.1 (2010): 1-136.

[6] Ferrero, Valter, and S. Camatel. "Optical phase locking techniques: an overview and a novel method based on single side sub-carrier modulation." Optics express 16.2 (2008): 818-828. 